\title{
Elastic Scattering, Pion Production, and Annihilation into Pions in Antiproton-Proton Interactions at $5.7 \mathrm{GeV} / \mathrm{c}$.
}

\author{
K. Böckmann, B. Nellen, E. Paul and B. Wagini
}

Physikalisehes Institut der Universität Bonn und $K F$ A Jülich - Bonn

I. Borecka, J. Diaz, U. Heeren, U. Liebermfister, E. L Johrmakn, E. RauboLd, P. Sodorng and S. Wolfe

Physikalisehes Staatsinstitut, II. Institut für Experimentalphysik und DESY - Hamburg

J. Kidd, L. Mandelli, L. Mosca, V. Pelosi, S. Ratti and L. Tallone

Istituto di Fisica e Sezione I.N.F.N. - Milano

(Nuovo Cimento, $42 \mathrm{~A}, 954(1966)$ )

Unfortunately two wrong figures have been inserted in this paper. Hereunder we print the correct figures with their captions:
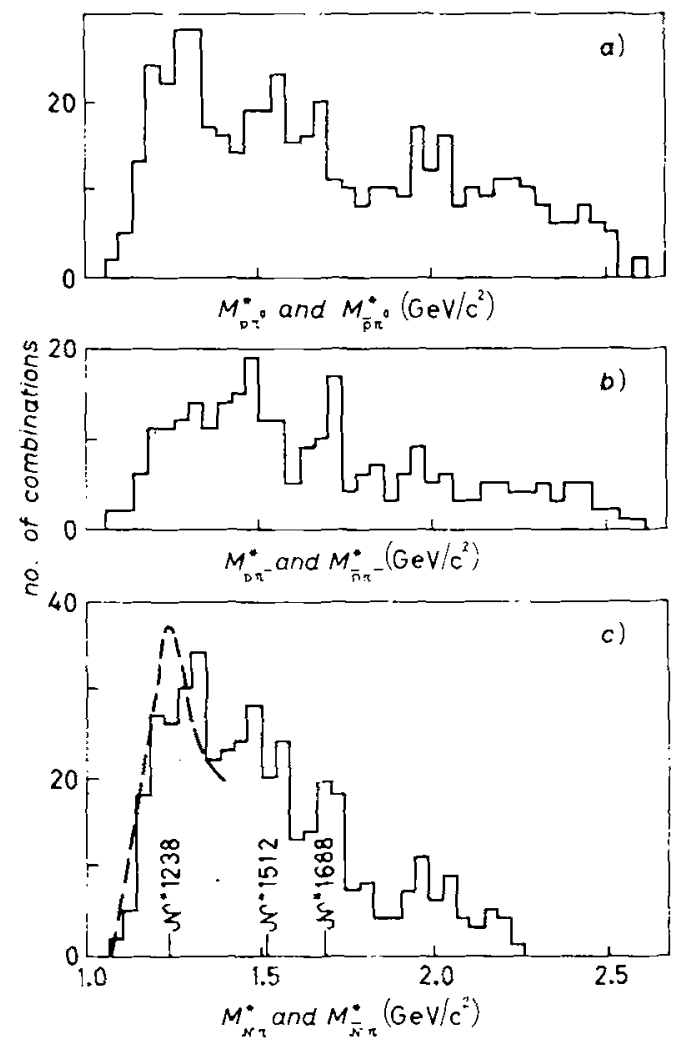

Fig. 2. - Effective-mass distributions for $(\mathcal{N} \pi)$ and $(\overline{\mathcal{N}} \pi)$ systems in singlepion production: a) effective-mass distribution of the systems $\left(p \pi^{0}\right)$ and $\left(\overline{\mathrm{p}} \pi^{0}\right)$ for 243 events of the type $\overline{\mathrm{p}} \mathrm{p} \pi^{0}$; b) effective-mass distribution of the systems $\left(p \pi^{-}\right)$and $\left(\bar{n} \pi^{-}\right)$for 138 events of the type $\left.\mathrm{p} \overline{\mathrm{n}} \pi^{-} ; c\right)$ effective mass distribution of the systems $(\mathcal{N} \pi)$ and $(\overline{\mathcal{N}} \pi)$ for 421 combinations of events of either type selected imposing the restriction for the four-momentum transfer squared between the initial (anti-) proton and final (anti-) nucleon-pion system, the mass of which is plotted, to be less than $0.3(\mathrm{GeV} / \mathrm{c})^{2}$. The curve is the OPEM prediction (with form factors) in the region of the $\left(\frac{3}{2}, \frac{3}{2}\right)$ isobar calculated with the same restrictions. 
Fig. 18. - Distributions of the square of the missing masses. a) Experimental distributions of $M M^{2}$ for 225 annihilation events all ambiguous between the two hypotheses $\left(2 \pi^{+} 2 \pi^{-} \pi^{0}\right)$ and $\left(2 \pi^{+} 2 \pi^{-} n \pi^{0}\right), n \geqslant 2$. b) $M M^{2}$ distribution for 129 faked events of the type $\left(2 \pi^{+} 2 \pi^{-} \pi^{0}\right)$ all found ambiguous with the hypothesis $\left(2 \pi^{+} 2 \pi^{-} n \pi^{0}\right)$, $n \geqslant 2$. c) $M M^{2}$ distribution for 132 faked events of the type $\left(2 \pi^{+} 2 \pi^{-} 2 \pi^{0}\right)$.

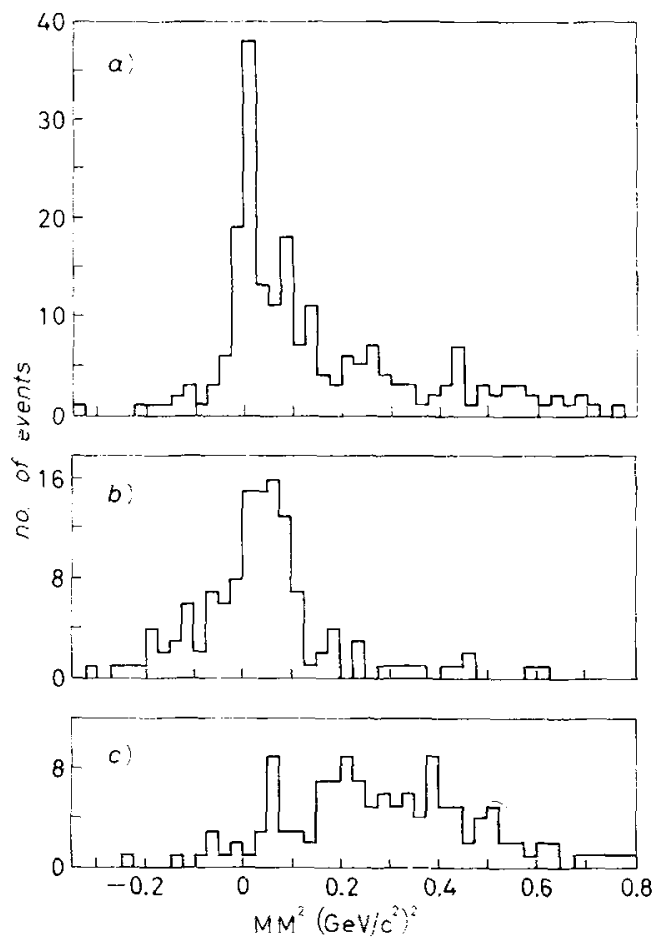

Please also note the following corrections:

in the Summary

$$
\begin{array}{ll}
\text { instead of } \sigma_{\text {annihil }}=(22.5 \pm 2.0) \mathrm{mb} & \text { read } \sigma_{\text {annihi1 }}=(22.0 \pm 2.0) \mathrm{mb}, \\
\text { instead of } \sigma_{\text {ine1 }}=(24.8 \pm 2.0) \mathrm{mb} & \text { read } \sigma_{\text {inel }}=(25.3 \pm 2.0) \mathrm{mb} ;
\end{array}
$$

in Table II on p. 959, lines 24 and 25 ,

insteat of 24.8 and 22.5

read 25.3 and 22 , respectively;

on p. 968 , line 17

instead of $\cos \delta \varphi=$

$\operatorname{read} \cos \varphi=$. 